\title{
Medical thoracoscopy removal of a self-expandable metallic stent migration into pleural cavity
}

\author{
Wen-Chien Cheng ${ }^{1,2,3}$, Biing-Ru Wu ${ }^{1,2}$, Chia-Hung Chen ${ }^{2,4,5,6}$, Wei-Chih Liao ${ }^{1,2,3}$, Chih-Yen Tu ${ }^{7}$ \\ ${ }^{1}$ Division of Pulmonary and Critical Care Medicine, Department of Internal Medicine, China Medical University Hospital, Taichung; ${ }^{2}$ School \\ of Medicine, ${ }^{3}$ Department of Internal Medicine, Hyperbaric Oxygen Therapy Center, ${ }^{4}$ Department of Respiratory Therapy, ${ }^{5}$ Graduate Institute \\ of Clinical Medical Science, China Medical University, Taichung; ${ }^{6}$ Taiwan Clinical Trial Consortium for Lung Diseases (TCoC), Taichung; \\ ${ }^{7}$ Department of Life Science, National Chung Hsing University, Taichung \\ Correspondence to: Chih-Yen Tu, MD. Department of Internal Medicine, China Medical University Hospital, No. 2, Yude Road, Taichung. \\ Email: chesttu@gmail.com.
}

\begin{abstract}
Pleuroscopy, also known as medical thoracoscopy (MT), is an important tool for pleural disease management. Removal of foreign body (FB) by MT was reported in a few reports because it can identify the position of a FB in the pleural cavity and can be used for its urgent removal. In this study, we describe the case of a male patient with a ruptured lung abscess and a rare complication of self-expandable metallic stent (SEMS) migration into the pleural cavity via a bronchopleural fistula after the management of obstructive airway disease. An experienced pulmonologist successfully removed the SEMS from the pleural cavity using semi-rigid pleuroscopy under local anesthesia and conscious sedation in a bronchoscopy suite.
\end{abstract}

Keywords: Self-expandable metallic stent (SEMS); migration; pleuroscopy; medical thoracoscopy (MT); lung abscess; bronchopleural fistula

Submitted Jan 29, 2018. Accepted for publication Mar 29, 2018.

doi: $10.21037 /$ jtd.2018.05.33

View this article at: http://dx.doi.org/10.21037/jtd.2018.05.33

\section{Introduction}

Pleuroscopy, or medical thoracoscopy (MT), is commonly used to diagnose unknown exudative pleural effusions, pleural drainage, and pleurodesis. MT can be divided into rigid and semi-rigid pleuroscopy. Rigid pleuroscopy can provide a larger window for the operator, who can use more instruments concurrently. There is a paucity of reports on the removal of foreign body (FB) by MT $(1,2)$. Selfexpandable metallic stent (SEMS) has been widely used for the treatment of benign or malignant airway disease. The complication of SEMS migration into the pleural cavity via bronchopleural fistula is rare reported. Here, we report a case with ruptured lung abscess having SEMS migration into the pleural cavity via bronchopleural fistula after obstructive airway disease management and its successful removal from the pleural cavity by semirigid pleuroscopy.

\section{Case presentation}

A 60-year-old man with right lower-lobe squamous cell lung carcinoma who had received concurrent chemoradiotherapy (CCRT) and chronic obstructive lung disease (COPD) showed slow disease progression without further anticancer therapy. He presented with gradually progressive dyspnea and fever. A chest radiograph showed right-side "whiteout" with signs of volume loss compared to previous image (Figure 1). Computed tomography (CT) of the thorax revealed a right lower lobe atelectasis and a lung abscess, which may have resulted from tumor necrosis (Figure 2A). There was no improvement in his symptoms even after 1 week of antibiotic treatment. The management of the lung abscess included adequate drainage and appropriate antibiotics. Endobronchial obstruction caused due to squamous cell lung carcinoma may play a role in the failure of the abscess cavity to drain spontaneously. Hence, 
bronchoscopy was performed to rule out endobronchial obstruction as a cause of poor drainage of lung abscess and atelectasis, an endobronchial tumor was observed in the right intermediate bronchus (Figure 2B). Electrocautery and SEMS insertion were performed to relieve the obstructive bronchus, which could improve dyspnea and lung abscess drainage. During the procedure, despite being under conscious sedation (using midazolam), the patient was agitated because of discomfort, which led to malposition of SEMS. The SEMS was placed too distally, and we were unable to visualize it through bronchoscopy. After 5 days of the procedure, a CT scan of the chest confirmed SEMS migration into the ruptured lung abscess and the pleural cavity, causing secondary empyema via the bronchopleural fistula (Figure 3). A discussion with the thoracic surgeon confirmed that because of poor pulmonary function, the

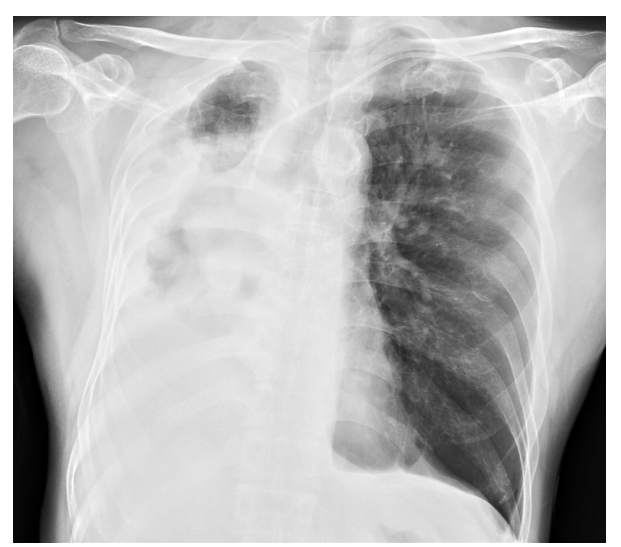

Figure 1 Chest radiograph showed right side "whiteout" with signs of volume loss. patient was not a good candidate for surgery under general anesthesia. Therefore, we decided to proceed with semirigid pleuroscopy to remove SEMS under local anesthesia (xylocaine) and sedation (using midazolam). Before the procedure, we used ultrasound to find a safe route and position of the SEMS (Figure 4). Under aseptic conditions, we made a skin incision and blunt dissection through the chest wall. A plastic trocar with a cannula was inserted across the chest wall into the pleural cavity, the trocar was removed, and semi-rigid pleuroscopy was performed. However, because of severe adhesion, the pleural cavity could not be easily examined. Fortunately, SEMS was found inside air-filled cavities within necrotizing lung tissue (Figure 5A). SEMS was gently grasped using forceps and successfully removed from the pleural cavity (Figure 5B,C) and pigtail catheter was placed for empyema and drainage of air leak. Thereafter, a new SEMS was correctly placed in the right intermediate bronchus.

After the procedure, the patient's dyspnea and fever disappeared and he was discharged under stable condition. Follow-up after a week showed that the patient was asymptomatic, and a chest $\mathrm{X}$-ray showed no SEMS migration (Figure 5D).

\section{Discussion}

The treatment of lung abscess included appropriate antibiotics and adequate drainage. Inadequate primary therapy of lung abscess may lead to the spread of lung abscess to pleural cavity via bronchopleural fistula. It is important to open the airway when the endobronchial obstruction is the cause of poor drainage of lung abscess.
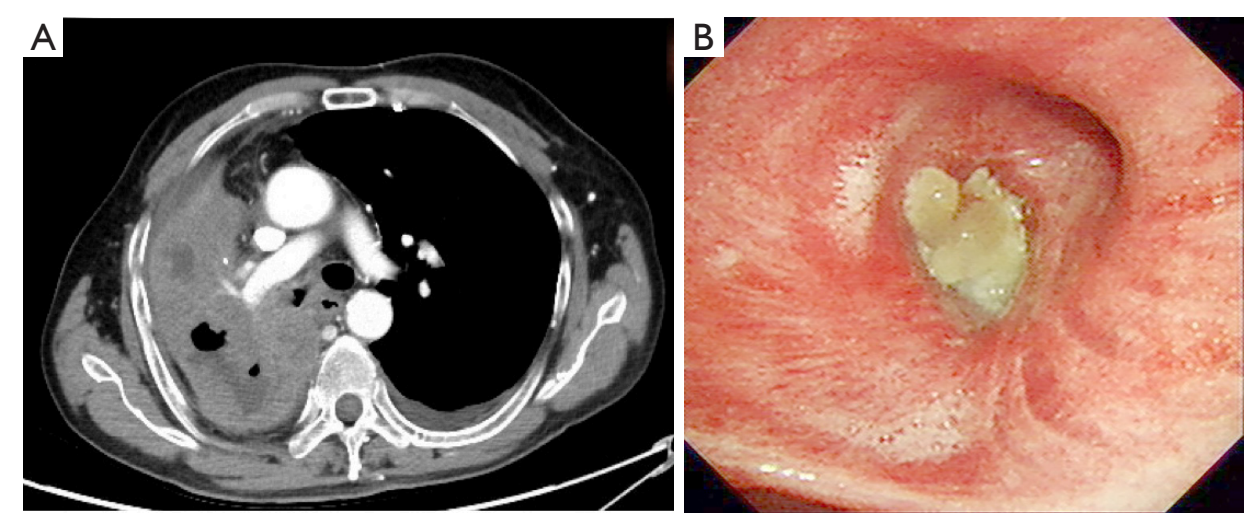

Figure 2 Chest tomography scan and bronchoscopic examination. (A) Computed tomography of thorax revealed right lower lobe lung abscess. (B) Bronchoscopy show an endobronchial tumor was observed in the right intermediate bronchus. 


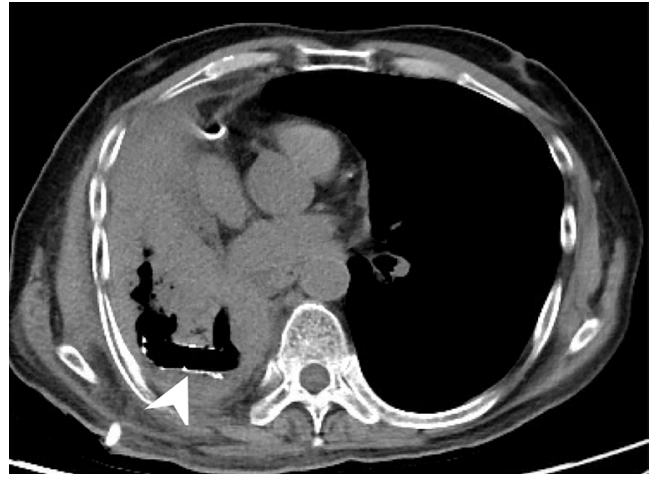

Figure 3 The migration of self-expandable metallic stent (SEMS) (arrow head) into the ruptured lung abscess and pleural cavity was confirmed by CT of chest.

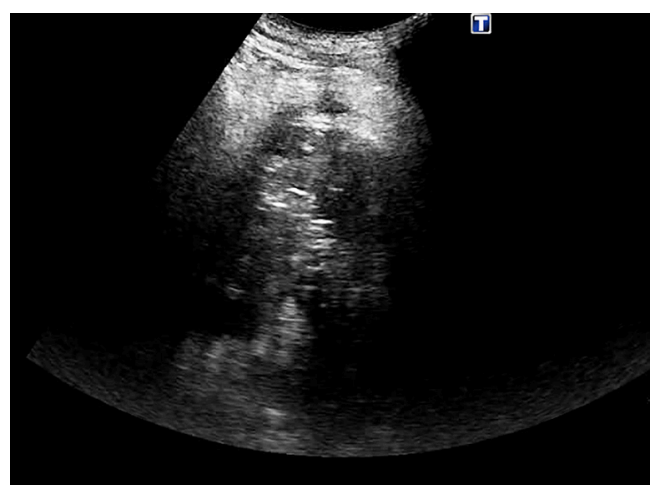

Figure 4 Chest ultrasound showed a hypoechoic lesion with typical pulmonary consolidation, irregular wall width, irregular luminal and outer margins.
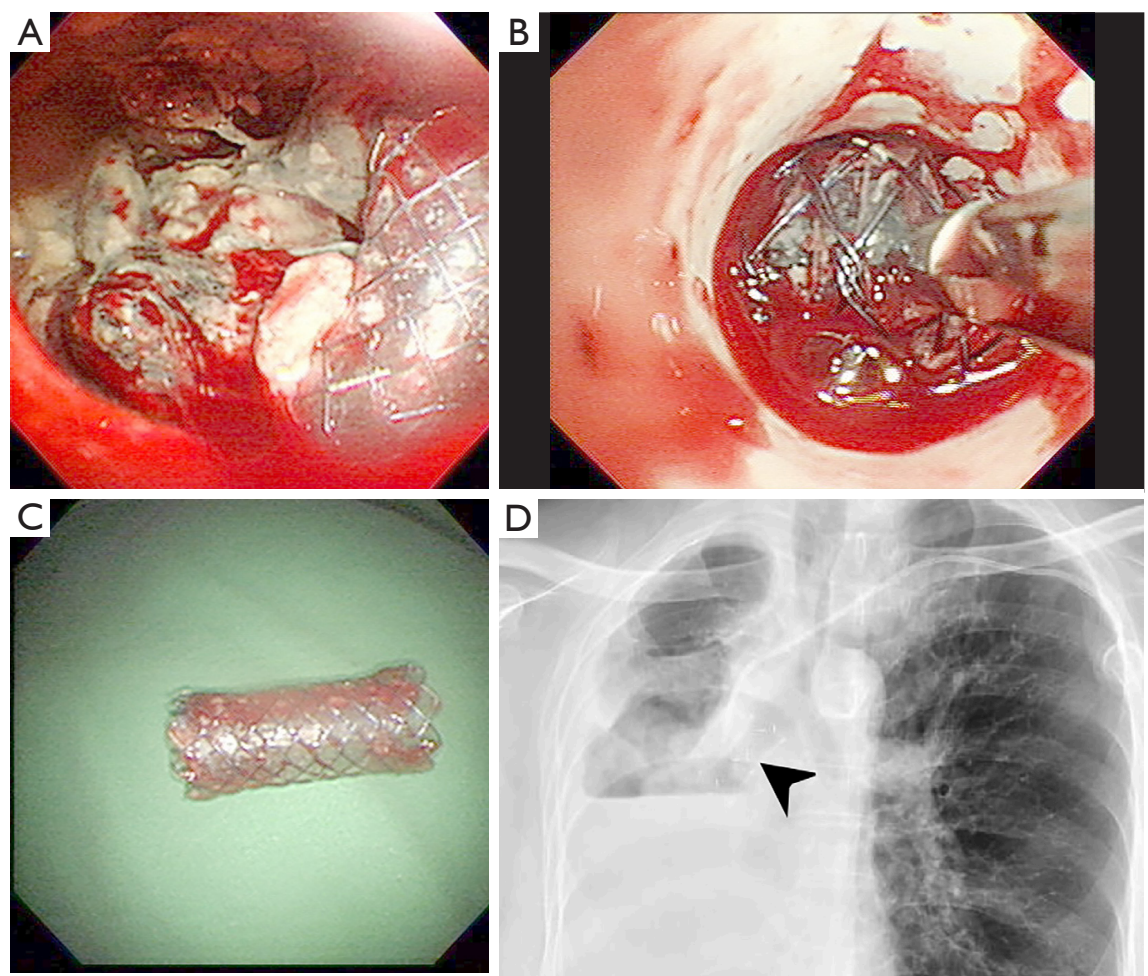

Figure 5 The process of removing and implantation self-expandable metal stent. (A) Self-expandable metallic stent (SEMS) was found in air-filled cavities within necrotizing lung tissue; $(B, C)$ the SEMS was grasped gently by forceps and removed from the pleural cavity successfully; (D) SEMS was placed at right intermediate bronchus correctly (arrow head) and without migration.

Interventional bronchoscopy with insertion of SEMS can relieve airway obstruction in benign and malignant airway diseases and its complications included granuloma formation, stent fracture, recurrent lumen stenosis, bacterial colonization, decreased mucociliary clearance, and migration $(3,4)$.

There has been an increase in the number of interventional bronchoscopy procedures performed in the bronchoscopy site, which often require deep levels of sedation by an anesthesiologist. In these advanced 
procedures, moderate sedation (midazolam and fentanyl) and spontaneous ventilation are associated with a high complication rate compared to that with deep sedation (propofol) or general anesthesia and volume-cycled ventilation (5). In our study, the patient became agitated during the procedure because of discomfort under moderate intravenous midazolam sedation. Deep sedation using a continuous infusion of propofol by an anesthesiologist may be an alternative choice, which can increase patient safety and comfort, thereby increasing the success rate of the procedure.

To our knowledge, SEMS migration into the pleural cavity vial bronchopleural fistula has never been reported and this was a first case of successful airway SEMS removal from the pleural cavity using semi-rigid pleuroscopy by an experienced pulmonologist. A literature review shows few reports on thoracoscopic removal of FBs from the pleural cavity $(1,2,6)$; a pleural aspiration needle was the most common FB in these reports. The removal of airway SEMS from pleural cavity by semi-rigid pleuroscopy has hardly been reported. Lochowski et al. reported a case of a patient with left main bronchial laceration with perforation of the right pleural cavity as a complication of dynamic stent insertion (7). This patient underwent thoracotomy for dynamic stent removal. Previous studies show that thoracotomy and video-assisted thoracoscopic surgery (VATS) under general anesthesia are mainly used for the management of intrapleural FBs after thoracic trauma or medical intervention, especially in the case of a large $\mathrm{FB}$, pleural adhesion, hemothorax, or vital organ injury (8). Pleuroscopy, or MT, is an important diagnostic and therapeutic tool in cases of undiagnosed pleural effusion, malignant pleural effusion, pneumothorax, pleural empyema, and hemothorax. It may be used as an alternative tool for the removal of an FB from the pleural cavity. A CT scan of the chest performed before the procedure can provide more information about the position of the $\mathrm{FB}$ and the condition of the pleural cavity. However, the FB can move from the CT scan to the time of MT, especially in a non-loculated pleural effusion. A chest ultrasound should also be done to find a safe route to the FB.

Compared to thoracotomy and VATS, pleuroscopy has several advantages. It can be performed by a pulmonologist under local anesthesia and conscious sedation in a bronchoscopy suite, the length of stay in the intensive care unit is less, and the cost of treatment is low $(9,10)$. In addition, a single port is sufficient to remove the $\mathrm{FB}$, and this leads to reduction of pain and quick recovery. Our study demonstrated that for patients who are unfit for thoracotomy or VATS because of poor lung function, after a multidisciplinary discussion, semi-rigid pleuroscopy is the most feasible procedure to remove SEMS from the pleural cavity.

\section{Conclusions}

In this study, we reported a rare complication of SEMS migration into the pleural cavity via bronchopleural fistula after obstructive airway disease management in a patient with ruptured lung abscess. Semi-rigid pleuroscopy under conscious sedation and local anesthesia was the best procedure for this patient because of poor lung function. The success of the procedure depends on proper patient selection based on lung function, multidisciplinary discussions, and an experienced pulmonologist. Deep level sedation was often required in interventional bronchoscopy procedure.

\section{Acknowledgements}

None.

\section{Footnote}

Conflicts of Interest: The authors have no conflicts of interest to declare.

Informed Consent: A waiver for consent was obtained from the IRB committee.

\section{References}

1. Narasimhan RL, Sehgal IS, Dhooria S, et al. Removal of Intrapleural Foreign Body by Medical Thoracoscopy: Report of Two Cases and a Systematic Review of the Literature. J Bronchology Interv Pulmonol 2017;24:244-9.

2. Gupta R, James P, Thangakunam B, et al. Medical thoracoscopic removal of a metal needle from the pleural space. BMJ Case Rep 2014;2014:bcr2014207035.

3. Chung FT, Chen HC, Chou CL, et al. An outcome analysis of self-expandable metallic stents in central airway obstruction: a cohort study. J Cardiothorac Surg 2011;6:46.

4. Zakaluzny SA, Lane JD, Mair EA. Complications of tracheobronchial airway stents. Otolaryngol Head Neck Surg 2003;128:478-88. 
5. Ost DE, Ernst A, Grosu HB, et al. Complications Following Therapeutic Bronchoscopy for Malignant Central Airway Obstruction: Results of the AQuIRE Registry. Chest 2015;148:450-71.

6. Tie ST, Wong JL, Kannan SK, et al. Pleuroscopic retrieval of a sewing needle from the pleural cavity under conscious sedation by a chest physician. J Bronchology Interv Pulmonol 2012;19:246-8.

7. Lochowski MP, Szlachcińska A, Kozak J. Left mainstem bronchial laceration with perforation to right pleural cavity as complication after dynamic stent insertion. Wideochir
Inne Tech Maloinwazyjne 2014;9:286-8.

8. Billeter AT, Druen D, Franklin GA, et al. Videoassisted thoracoscopy as an important tool for trauma surgeons: a systematic review. Langenbecks Arch Surg 2013;398:515-23.

9. Tassi GF, Tschopp JM. The centenary of medical thoracoscopy. Eur Respir J 2010;36:1229-31.

10. Parrish S, Browning RF, Turner JF Jr, et al. The role for medical thoracoscopy in pneumothorax. J Thorac Dis 2014;6:S383-91.

Cite this article as: Cheng WC, Wu BR, Chen CH, Liao WC, Tu CY. Medical thoracoscopy removal of a self-expandable metallic stent migration into pleural cavity. J Thorac Dis 2018;10(5):3054-3058. doi: 10.21037/jtd.2018.05.33 